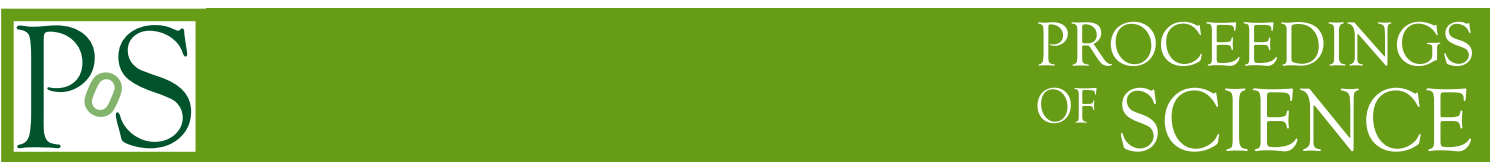

\title{
FRATs: a real-time search for Fast Radio Transients with LOFAR
}

\section{S. ter Veen*}

Radboud University Nijmegen (Department of Astrophysics)

E-mail: s.terveeneastro.ru.nl

\section{H. Falcke}

Radboud University Nijmegen (Department of Astrophysics), ASTRON

\section{R. Fender}

University of Southampton (School of Physics \& Astronomy)

\section{J.R. Hörandel}

Radboud University Nijmegen

\section{C.W. James}

Radboud University Nijmegen (Department of Astrophysics)

\section{S. Rawlings}

Oxford University

\section{P. Schellart}

Radboud University Nijmegen (Department of Astrophysics)

\section{B. Stappers}

University of Manchester (School of Physics \& Astronomy)

\section{R. Wijers}

University of Amsterdam

\section{M.Wise}

ASTRON

\section{P. Zarka}

Observatoire de Paris (LESIA) 
The radio sky is not steady on timescales below one second. Pulsars (including the rotating radio transients RRATs) and solar-system objects (e.g. solar flares, jupiter bursts, saturn lightning) give rise to sub-second pulses. Also in many known radiation processes coherent radiation can more easily occur at longer wavelengths, for which the size of the emitting region is comparable to the wavelength. This makes low frequency surveys ideally suited for the detection of new emission mechanisms caused by compact objects, such as white dwarfs, neutron stars and black holes. To detect as many of these Fast Radio Transients (FRATs) as possible, we are setting up a technique to detect and identify short single pulses with LOFAR in real-time, with unprecedented sensitivity in this frequency range, and excellent discrimination against terrestrial signals.

ISKAF2010 Science Meeting - ISKAF2010

June 10-14, 2010

Assen, the Netherlands

${ }^{*}$ Speaker. 


\section{Introduction}

With recently built instruments and the current state of computing power, the transient sky has opened up at many wavelengths, monitoring the sky continuously for outbursts. For example in $\mathrm{X}$-rays this is done for several years already with "all-sky" monitors built into satellites. They can alert the community of any interesting transient events, to trigger multi-wavelength observations. With LOFAR, the Low Frequency Array [1], a new instrument is added to this list with capabilities to monitor the low frequency radio sky at several timescales for shorter and longer variations. Pulsars, flare stars, planets and exoplanets are possible sources of these variations and, opening up a new window, thus far undetected phenomena may also be found, from coherent radiation processes in compact objects, such as neutron stars, white dwarfs and black holes. This is more likely at low frequency because the size of the emitting region for the currently known processes is comparable with these wavelengths. The LOFAR Transients Key Science Project is setting up the Radio Sky Monitor [2] to study the transient radio sky at time scales large then one second, while the Cosmic Ray Key Science Project develops techniques to image cosmic ray air showers [3], as well as lunar cascades caused by ultra high energy neutrinos and cosmic rays (NuMoon project[4]), at a timeresolution below 10 nanoseconds. With FRATs they combine their expertise to set up an observation mode for LOFAR to detect and identify sub-second single pulses of Fast Radio Transients in real-time.

To monitor the sky, the optimum is to view the whole sky all the time, because if an event happens and the telescope is not pointing in that direction, it will not be detected. This is also why there is still a huge discovery potential. Many events may have been missed, not only because people didn't analyse the data in this way, but also because there was no telescope pointing in the right direction at the right time. Only recently in off-line data analysis, people have been looking for single pulses, which lead to the discovery of RRATs (rotating radio transients) [5]. With the current state of LOFAR it is not possible to cover the complete sky all the time, but to come close to this, the largest field of view and observation time possible are needed. In the next section we describe how LOFAR can best be used to do both. Then we describe how to identify pulses with full LOFAR angular resolution and excellent RFI discrimination and finally give a status report of what has been achieved so far.

\section{Detecting and identifying short pulses with LOFAR}

LOFAR will consist of $42+$ stations with 96 dipoles each on baselines ranging from $100 \mathrm{~m}$ to a few hundred kilometers. It has different operation modes between 10-250 MHz. There are two basic modes of taking data with LOFAR at the lowest level. One is to use each station as a dish, the other option is to take data from single dipoles which is stored in buffers on the so-called Transient Buffer Boards (TBBs). After any of these two types of data is taken, the rest of the telescope is just software. That means you can correlate the data in any way you want, providing enough computing power is available. This opens up opportunities for parallel observations. For example, LOFAR is capable of taking imaging and beam-formed data at the same time. Beam-formed data is data from multiple stations added in the time domain, to maintain the high time resolution which is also required by pulsar observations. This can be done either coherently to maximize sensitivity, 
or incoherently to keep the wide field of view of a single station. The basic idea is to trigger on short pulses in incoherently beam-formed data parallel to any observation mode, to maximize sky coverage and exposure time, and then save the buffers from the TBBs to analyze the data in detail, as with this data the signal can be reconstructed in any direction.

\subsection{Detection algorithm}

Any detection mechanism has to deal with constraints set by the source and the instrument. In this case the pulses are dispersed with an unknown dispersion measure (DM) and pulse shape, the processing time is limited as the trigger has to run in real-time, and we must do this within the constrains of the hardware architecture. To deal with all this we use the following procedure:

1. Form an incoherent beam parallel to the current observation. This beam is split up into many small frequency channels $(0.7-24 \mathrm{kHz})$

2. Incoherently de-disperse the signal by adding frequency channels in the power domain with the appropriate time delays given by Eq. 2.1. This is done in multiple frequency bands due to the distributed data, and for different DM trials.

$$
\Delta t=4.15 \mathrm{~ms} \mathrm{DM}\left[\frac{1}{v_{2, \mathrm{GHz}}^{2}}-\frac{1}{v_{1, \mathrm{GHz}}^{2}}\right]
$$

3. Search for a pulse in each frequency band separately by determining the $\mathrm{S} / \mathrm{N}$ ratio in each time bin from the average and the standard deviation. In this step integration over multiple time bins is possible, to allow for different pulse shapes.

4. Run a coincidence trigger on pulses found in the previous step with a time delay between the bands (Fig. 1), again given by the DM through Eq. 2.1. If a coincidence is found between enough frequency bands a trigger message is send.

5. Identify whether the pulse is generated by RFI, a known object or an unknown object.

6. Store Transient Buffer Board data and/or alert the outside world.

The use of multiple frequency bands is not only required because of the distributed data, but also leads to less false positives. RFI can increase the power in one band and cause a trigger, but it is less likely that RFI causes a trigger in other frequency bands as well with the correct delay. Also the data from other DMs can be an indication that the pulse found is a false positive. This can be because a strong real signal should also appear in adjacent DM trials or because a signal is found with a DM of zero in some frequency bands, which is usually caused by RFI or instrumental errors.

\subsection{Pulse identification}

After a trigger is received, all that is known is that there was a pulse in the wide field of view from the station beam some time ago. Fortunately, LOFAR has a time machine because the data is buffered for each antenna in the Transient Buffer Boards. If the pulse is classified as worth investigating further, this TBB data will be dumped to hard disks and used for data analysis. As 


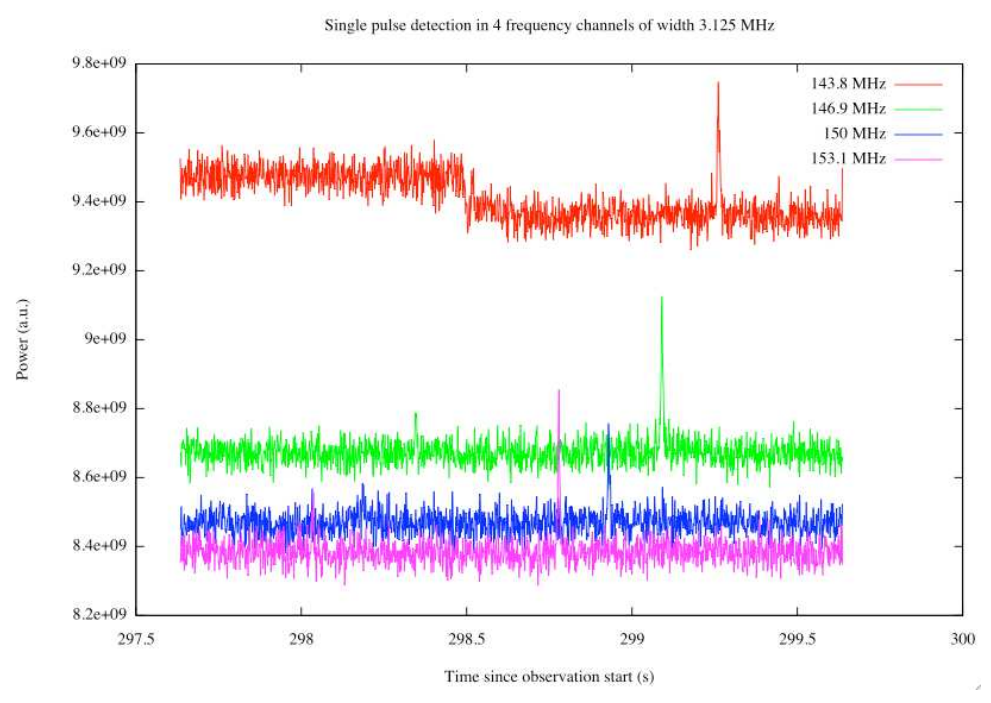

Figure 1: A pulse from B1508+55 detected in 4 frequency bands. Notice the delay the pulse has between the frequency bands. The drop in the upper band is most likely caused by an RFI channel switching of. This decreases the total power in this band.

the major part of the telescope is just software, and TBB data is the lowest level of data, this can be used to reconstruct the signal in any direction and to any distance! This is possible because the data has not yet been beam-formed at the station level. This opens up unique possibilities. For example an all sky map can be generated for the same instance in time Fig. (2). This can even be done up to the full angular resolution ( $\sim \operatorname{arcsec})$, as now a coherent beam can be formed. This also increases the $\mathrm{S} / \mathrm{N}$ ratio by a factor of 6 , when using 36 stations and allows for coherent dedisperion. Also the data can be beam-formed in the near field. With all these capabilities combined, the source direction can be found, even if it initially entered through a side-lobe, and the distance can be determined to check if the signal is human made RFI or comes from natural terrestrial sources such as lightning. This is a huge advantage over a single dish telescope as with these features it would immediately be clear if events like the Lorimer burst [6], a $30 \mathrm{Jy}$ pulse with a duration of $5 \mathrm{~ms}$ and a DM of $375 \mathrm{pc} \mathrm{cm}^{-3}$, would be extra-galactic or not even extra-terrestrial. Disadvantages are: the large data size ( $48 \mathrm{~GB} / \mathrm{station})$, the time to dump the data, and the limited time (1.3 $\mathrm{s}$ at full resolution) and therefore bandwidth of a pulse available in the buffer due to dispersion. The latter can be increased by trading bandwidth for time in the buffer or increasing the memory of the TBBs.

\section{Synergy with other LOFAR projects}

There is a large synergy with other LOFAR projects. Triggering on a signal to dump TBB data is also done in the Cosmic Ray Air Shower[3] and NuMoon[4] projects. They intend to discover high energy cosmic rays and ultra high energy cosmic rays and neutrinos respectively. The only difference is in the way a trigger is generated, the amount of data stored to disk (only $1 \mathrm{~ms}$ for both projects) and the dispersion of the signal. But the same analysis tools can be used for the TBB data 


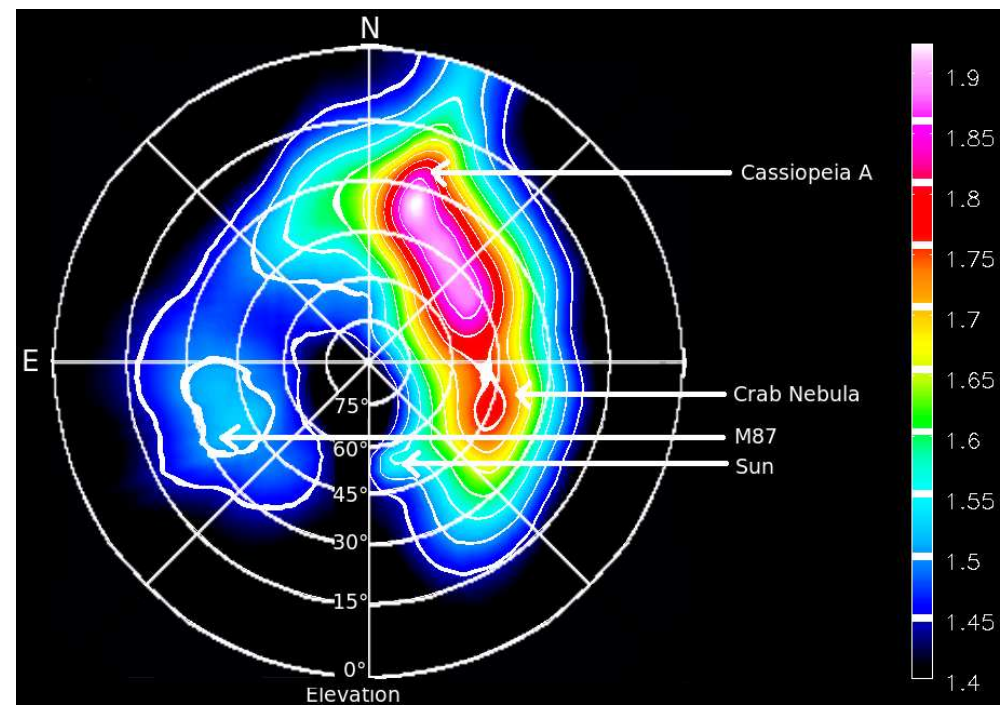

Figure 2: An instantaneous all-sky map between 30-85 MHz, using 43 antennas from LOFAR station CS302. Integration time $5 \mathrm{~ms}$. With more stations the resolution will increase.

to generate images. To identify an outburst in an image, the pipeline of the Radio Sky Monitor from the Transients Key Science Project can be used [7].

\section{Status}

Currently the trigger algorithm can run multiple DM trials at the same time. With it we have been able to detect single pulses of six pulsars which were used as test sources. These pulsars are B0329+54, B2217+47, B1919+21, B1508+55, B1133+16 and the Crab pulsar and have been triggered on (apart from B1508+55) requiring a 4 sigma detection in at least 7 bands with a bandwidth of $2 \mathrm{MHz}$, using 10 full LOFAR stations, between 143 and $163 \mathrm{MHz}$. In real-time mode we are able to dump TBB data on a received trigger. We have not yet detected a pulse in dumped TBB data, because either the signal to noise ratio was too low or there are still some errors in our analysis software.

\section{Summary}

By combining the large field of view of a single LOFAR station with the increased sensitivity given by incoherently combining multiple stations at high time resolution, we are able to trigger on dispersed single pulses in real time parallel to other observations, thereby maximizing the detection probability. After this, buffers from each antenna can be dumped and analyzed to find the direction and distance of the pulse and thereby identifying the object that generated it, where it can distinguish between terrestrial and astrophysical sources. Also a message for multi-wavelength followup can be send out. With this method FRATs adds low radio frequencies to the list of wavelengths for which a significant part of the sky can be monitored for sub-second single pulses with unprecedented sensitivity and large discovery potential for new objects and new emission processes. 


\section{References}

[1] LOFAR, www.lofar.org

[2] Fender, R., Wijers, R., Stappers, B., \& The LOFAR Transients Key Science Project 2008, arXiv:0805.4349

[3] Horneffer, A. et al, 2008, arXiv:0903.2398

[4] O. Scholten et al., Astropart. Phys. 26, 219 (2006).

[5] McLaughlin, M. A. et al. Nature 439: 817-820 (2006)

[6] Lorimer, D.R., Bailes, M., McLaughlin, M.A., Narkevic, D.J. \& Crawford, F. 2007, Science, 318, 777

[7] Swinbank, J. et al. A transient detection and monitoring pipeline for LOFAR, Proceedings of "Bursts, Pulses and Flickering: wide-field monitoring of the dynamic radio sky". 12-15 June 2007, Kerastari, Tripolis, Greece 